\title{
Polycythemia Vera
}

\section{FURTHER IN VITRO STUDIES OF HEMATOPOIETIC REGULATION}

\author{
John W. Adamson, Jack W. Singer, Pat Catalano, Scott Murphy, \\ Nancy Lin, Laura Steinmann, Connie Ernst, and Philip J. Fialkow, \\ Sections of Hematology, Oncology and Medical Genetics, Medical Service, \\ Veterans Administration Medical Center and University of Washington, \\ Seattle, Washington 981.95; The Cardeza Foundation, Thomas Jefferson \\ University, Philadelphia, Pennsylvania 19107
}

\begin{abstract}
A B S T RACT Further in vitro studies of hematopoietic regulation were carried out in two patients with polycythemia vera who were also heterozygotes $\left(\mathrm{Gd}^{\mathrm{B}} / \mathrm{Gd}^{\mathrm{A}}\right)$ for glucose-6-phosphate-dehydrogenase (G-6-PD). While only G-6-PD type A was detectable in circulating erythrocytes, granulocytes and platelets, cultures of peripheral blood and marrow from one patient revealed a substantial number of G-6-PD type $B$ erythroid burst-forming units (BFU-E) and granulocyte/macrophage colony-forming units. Detailed analysis demonstrated: (a) where detectable, normal BFU-E and granulocyte/macrophage colony-forming units were found with similar frequencies; $(b)$ the same frequencies for normal progenitors characterized cultures of peripheral blood and marrow; $(c)$ inhibition of normal erythroid differentiation between BFU-E and the more mature erythroid colony-forming unit; $(d)$ a decline in the prevalence of normal colony-forming units with time, suggesting that disease progression is associated with further suppression of normal hematopoiesis by products of the abnormal clone.
\end{abstract}

\section{INTRODUCTION}

Previous studies have demonstrated that polycythemia vera is a disease arising in a pluripotent stem cell which, at least at the time of evaluation, appears to be clonal (1). These conclusions were reached by studies in patients who had naturally occurring cellular mosaicism. The marker system employed was the isoenzymes of glucose-6-phosphate dehydrogenase (G-

Presented in part at the 21st Annual Meeting of the American Society of Hematology 1-4 December 1978, and published in abstract form in 1978. Blood. 52(Suppl. 1): 199.

Received for publication 13 March 1980 and in revised form 14 August 1.980.
6-PD). ${ }^{1}$ The structural gene for G-6-PD is on the $\mathrm{X}$ chromosome and, because it undergoes $\mathrm{X}$ chromosome inactivation, in a female heterozygous for G-6-PD only one of the two isoenzymes is synthesized in a single cell. In hematologically normal women, the circulating blood elements have isoenzyme ratios similar to those found in skin fibroblasts (2). In contrast, G-6-PD heterozygotes with several myeloproliferative disorders, including polycythemia vera (1), chronic myelogenous leukemia $(3,4)$, and agnogenic myeloid metaplasia with myelofibrosis (5), had circulating blood elements that contained only a single isoenzyme. Thus, these diseases represent stem cell disorders of presumed clonal origin.

In earlier studies, we cultured hematopoietic cells for both erythroid and granulocytic colony growth and determined that progeny of presumed normal stem cells persisted in marrows of patients with polycythemia vera (6). These hematopoietic colonies could be individually harvested from the semisolid medium and subjected to electrophoresis for G-6-PD type. By this analysis, the gene products of individual cells were clonally amplified, thus affording the opportunity to determine the isoenzyme type of the single progenitor for each colony. The results of the initial studies showed that a high percentage of apparently normal colony-forming cells could be detected in the marrow of such patients (6).

Although it is likely that most colonies assayed in the original studies were derived from primitive erythroid progenitors (BFU-E; erythroid burst-forming unit), a clear distinction was not made between these

'Abbreviations used in this paper: BFU-E, erythroid burst-forming unit; CFU-C, granulocyte/macrophage colony-forming unit; CFU-E, erythroid colony-forming unit; ESF, erythropoietin; G-6-PD, glucose-6-phosphate dehydrogenase. 
and more mature erythroid progenitors (CFU-E; erythroid colony-forming unit). It is believed that BFU$\mathrm{E}$ give rise to CFU-E and are more closely related to pluripotent stem cells $(7,8)$. In addition, because no source of colony-stimulating factor had been added to the cultures, granulocyte/macrophage colony forming cells (CFU-C) had not been grown under optimal conditions.

We have restudied the two patients with polycythemia vera in greater detail to answer the following questions: (a) Do isoenzyme patterns vary in colonies derived from erythroid progenitors of different stages of maturation? ( $b$ ) Are the frequencies of "normal" granulopoietic and erythroid progenitors similar? $(c)$ Are these frequencies similar in peripheral blood and marrow? $(d)$ Is there a decrease in the detected frequency of normal progenitors over time?

\section{METHODS}

Subjects. Clinical details of the patients were reported previously (1). Neither the modes of treatment nor clinical courses had changed significantly in the interval since 1976. Patient 1 had been treated intermittently with chlorambucil and occasional phlebotomy, but had received no chemotherapy within 2 mo of these studies. Patient 2 continues to be treated with phlebotomy only. Patient 1 was hospitalized in the Clinical Research Center at the University of Washington Hospital in February 1978. Marrow and peripheral blood were obtained on two occasions with the informed consent of the patient. Laboratory values at the time of study were hematocrit, 46\%; hemoglobin, $15.5 \mathrm{~g} / \mathrm{dl}$; leukocyte count, $8.3 \times 10^{\%} /$ liter; and platelet count, $2.6 \times 10^{11 /} /$ iter. Peripheral blood, collected and shipped in preservative-free heparin, was also obtained for culture in October 1978 and in January 1979, and flown at ambient temperature to Seattle, Wash.

Marrow and peripheral blood specimens were obtained in preservative-free heparin from patient 2 in December 1977, and peripheral blood was obtained again in December 1978. These specimens were also flown at ambient temperature to Seattle. A maximum of $12 \mathrm{~h}$ elapsed between sampling time and the time that cultures were established. Laboratory values for patient 2 at the time of marrow sampling were hematocrit, $41.5 \%$; leukocyte count, $35.4 \times 10^{9} /$ liter; and platelet count, $5.53 \times 10^{11 /} /$ liter. Hematologic values in December 1978 were similar.

Cell culture. Buffy coat marrow cells were cultured as previously described $(6,9)$. Peripheral blood was separated over Ficoll-Hypaque (Pharmacia Fine Chemicals, Inc., Piscataway, N. J.), interface cells collected, washed three times in alpha-medium containing $5 \%$ fetal calf serum, and cultured at concentrations of $0.75-1.0 \times 10^{5}$ cells $/ \mathrm{ml}$. For erythroid colony growth, cells were cultured in the presence of 0.25 and $5.0 \mathrm{U} / \mathrm{ml}$ of sheep plasma erythropoietin (ESF; Connaught Laboratories, Ontario, Canada). Growth of granulocyte/macrophage colonies was stimulated by the addition to separate cultures of a phytohemagglutinin-stimulated conditioned medium prepared from human peripheral blood mononuclear cells (9).

Colonies were enumerated, individually harvested from the methylcellulose using microcapillaries, and then subjected to electrophoresis for determination of G-6-PD isoenzyme type at times appropriate for the various progenitor types (days 6, 10-12, and 13-15 for CFU-E, BFU-E, and CFU-C-
TABLE I

Bone Marrow Cultures in Polycythemia Vera (Patient 1)

\begin{tabular}{lrcc}
\hline \multicolumn{1}{c}{ Colony type } & Patient 1 & Patient 2 & Nornal subjects* \\
\hline & \multicolumn{3}{c}{ colonies $/ 10^{5}$ nucleated cells plated } \\
CFU-E & & & \\
No added ESF & 6.2 & 7.1 & \\
5.0 U ESF & 64.4 & 41.7 & $91 \pm 20^{*}(n=22)$ \\
BFU-E & & & - \\
0.25 U ESF & 3.0 & 2.5 & $28 \pm 8 \quad(n=22)$ \\
5.0 U ESF & 16.2 & 15.1 & $80 \pm 40(n=46)$ \\
CFU-C & 50.3 & 29.6 & 80 \\
\hline
\end{tabular}

$* \pm \mathrm{SD}$.

derived colonies, respectively). Greater than $90 \%$ of all colonies tested were analyzable from growing cultures. To preclude selection bias, all the colonies identifiable at $\times 25$ magnification were harvested for analysis. If there were too many colonies, $1 / 4$ or $1 / 8$ of the culture dish was marked off and all colonies within the sector were harvested.

Statistical analysis. Estimates of the frequency of normal colony-forming cells were based on the fact that each subject had approximately equal amounts of $A$ and $B$ isoenzyme activities in skin (1). Consequently, for every presumed normal B-type colony detected, one of the A-type colonies is also normal. The overall estimate of the percentage of normal progenitors is twice that of the percentage of B-type colonies. Statistical significance was tested by chi-square analysis.

\section{RESULTS}

\section{Colony numbers}

The numbers of the various colony types are shown in Table I. As was observed previously, there was an ESF dose-dependent increase in the numbers of detectable CFU-E and BFU-E.

\section{Isoenzyme analysis}

Analysis of direct preparations. Only G-6-PD type A was found in peripheral blood erythrocytes, granulocytes, and platelets and in marrow buffy coat cells from both patients.

Isoenzyme patterns in erythroid and granulocytic colonies. Only 1 of $47 \mathrm{CFU}-\mathrm{E}$-derived colonies grown at 5.0 U ESF added to marrow cultures of patient 1 showed B-type G-6-PD and thus presumably arose from a normal progenitor (Table II). Although none of the erythroid bursts detected at $0.25 \mathrm{U} \mathrm{ESF} / \mathrm{ml}$ were type B, significant numbers which manifested type B enzyme were found in cultures containing the higher concentration of ESF. For CFU-E and those BFU-E that formed colonies at low concentrations of ESF, the estimated frequency of normal progenitors was $<5 \%$. At the higher concentration of ESF, the estimated frequency of normal progenitors was $20 \%$. A similar 
percentage of apparently normal CFU-C was also detected. Thus, for patient 1 , the data suggest that erythroid differentiation is dampened somewhere in the sequence between BFU-E having higher ESF requirements for growth and CFU-E and BFU-E requiring lower concentrations of ESF.

No presumably normal (type B) erythroid or granulocytic colonies were detected in cultures from patient 2 . This contrasts with the results of the study done 2 yr previously.

Comparison of the prevalences of normal CFU-C and BFU-E in peripheral blood and marrow. There were no significant differences in the prevalence of normal CFU-C or BFU-E (Table III). For this analysis, all data derived for BFU-E grown with 5.0 U ESF were combined.

\section{Ratio of CFU-C to BFU-E in the neoplastic and residual normal clones}

By applying the estimated percentages of normal colony-forming cells of various types to the absolute numbers of colonies detected, it is possible to calculate the ratios of CFU-C to BFU-E both in the abnormal clone and in the residual normal clones. The ratio derived for the polycythemia vera clone in patient 1 was 3.2 and in patient 2 it was 2.0. The ratio of CFU-C to BFU-E in residual normal clones detected in patient 1 was 2.7 , similar to the value for the abnormal clone. The ratio derived from 13 control subjects was 2.9 $(\mathrm{SE} \pm 1.3) .{ }^{2}$ Thus, the ratios of CFU-C to BFU-E in the abnormal clones did not differ significantly from ratios derived from normal subjects.

\section{Sampling variability}

To determine the variability due to sampling, specimens for culture were obtained from patient 1

${ }^{2}$ J. W. Adamson and J. W. Singer. Unpublished observations.

TABLE II

G-6-PD Analysis of Erythroid Colonies

\begin{tabular}{ccccc}
\hline & & \multicolumn{2}{c}{$\begin{array}{c}\text { Isoenzyme } \\
\text { type }\end{array}$} & \\
\cline { 3 - 4 } Patient & & A & B & Percent normal \\
\hline \multirow{2}{*}{1} & CFU-E & & & \\
& 5.0 U ESF & 46 & 1 & $<5$ \\
& BFU-E & & & $<5$ \\
& 0.25 U ESF & 37 & 0 & 20 \\
& 5.0 U ESF & 128 & 14 & $<5$ \\
& CFU-E & 44 & 0 & $<5$ \\
\hline & BFU-E & & & \\
& 5.0 U ESF & 25 & 0 &
\end{tabular}

TABLE III

G-6-PD Analysis of Colony-Forming Units from Marrow and Peripheral Blood (Patient 1)

\begin{tabular}{lrrr}
\hline & \multicolumn{2}{c}{ Isoenzyme type } & \\
\cline { 2 - 3 } & \multicolumn{1}{c}{ A } & B & Percent nomal \\
\hline Marrow & & & \\
$\quad$ BFU-E & 205 & 21 & 19 \\
CFU-C & 92 & 8 & 16 \\
Peripheral blood & & & \\
$\quad$ BFU-E & 59 & 6 & 18 \\
CFU-C & 69 & 8 & 21 \\
\hline
\end{tabular}

several days apart from opposite posterior iliac crest sites. Of 142 erythroid bursts analyzed from cultures obtained from the left side, 14 typed as B (20\% normal). The observations from cultures from the opposite side were 77 and 7 ( $17 \%$ normal). This analysis found no significant differences in the estimates of the percentage of normal progenitors based on marrow site.

\section{Change in prevalence of normal colony-forming cells with time}

A significant decline in the prevalence of normal colony-forming cells was found between the original study and the current studies (Table IV). These findings were confirmed in January 1979, at which time none of 11 peripheral blood BFU-E-derived colonies analyzed for patient 1 typed as B, indicating that the incidence of normal committed progenitors had declined to $<10 \%$. Additional studies from patient 2 failed to detect any normal BFU-E or CFU-C-derived colonies under standard culture conditions.

\section{DISCUSSION}

In patients with myeloproliferative diseases, we took advantage of naturally occurring cellular mosaicism and assessed disease progression and mechanisms by which abnormal clones influence normal hematopoiesis. The initial studies demonstrated a notable frequency of presumably normal erythroid and granulocyte/macrophage colonies (6). This led to the conclusion that the differentiation of normal marrow committed stem cells in such patients was somehow suppressed. The mechanism of suppression of erythropoiesis was not due to decreased ESF stimulation, since not only were the hemoglobin concentrations and hematocrits of the two patients normal at the time of study, but ESF excretion was normal in patient 1 as measured by the exhypoxic polycythemic mouse assay (6).

In the present study, we analyzed in greater detail the level at which normal erythroid differentiation is in- 
TABLE IV

Decline in the Frequency of Normal Erythroid Bursts with Time

\begin{tabular}{|c|c|c|c|c|c|c|c|}
\hline \multicolumn{4}{|c|}{ Patient 1} & \multicolumn{4}{|c|}{ Patient 2} \\
\hline \multirow[b]{2}{*}{ Date } & \multicolumn{2}{|c|}{$\begin{array}{c}\text { Isoenzyme } \\
\text { type }\end{array}$} & \multirow[b]{2}{*}{ Percent normal } & \multirow[b]{2}{*}{ Date } & \multicolumn{2}{|c|}{$\begin{array}{c}\text { Isoenzyme } \\
\text { type }\end{array}$} & \multirow[b]{2}{*}{ Percent norma } \\
\hline & A & B & & & A & $\mathrm{B}$ & \\
\hline 1976 & 26 & 10 & 56 & 1976 & 30 & 8 & 42 \\
\hline February 1978 & 205 & 21 & 19 & December 1977 & 49 & 0 & $<5$ \\
\hline January 1979 & 11 & 0 & $<10$ & December 1978 & 22 & 0 & $<5$ \\
\hline
\end{tabular}

hibited. In the hierarchy of erythropoietic differentiation, BFU-E are thought to be closely related to pluripotent stem cells and give rise to more mature colonyforming cells, the CFU-E. Significant numbers of normal colonies were detected only among BFU-E requiring higher concentrations of ESF. Therefore, normal erythroid differentiation appears to be reduced somewhere between primitive BFU-E and the more mature CFU-E.

One explanation for the negligible frequency of normal CFU-E is that there is an exaggerated amplification of CFU-E numbers within the neoplastic clone. However, the CFU-E:BFU-E ratio from the abnormal clone was $4.8: 1$, a value within the range obtained by our laboratory for 39 normal marrows (mean $2.8 ; \mathrm{SD} \pm 1.6$ ). ${ }^{3}$ Thus, the marked reduction in the estimated percentage of normal CFU-E compared to BFU-E (ratio of 0.8 ) could not be accounted for by a dramatic increase in the number of CFU-E within the neoplastic clone. The mechanism by which the expected amplification from normal BFU-E to CFU-E is reduced in vivo is not known, but it possibly involves cellular interactions.

As has been reported previously in polycythemia vera, significant numbers of CFU-E-derived colonies will form in marrow cultures containing no added ESF. The number of these so-called "endogenous" colonies varies from patient to patient and with time in the same patient. The possibility has been raised that hypersensitivity to ESF in culture identifies members of the neoplastic clone and can be used to determine its limits (10). The results of this study suggest otherwise.

As shown in Table II, when data from both patients are combined, only 1 of 91 CFU-E-derived colonies typed as $\mathrm{B}$ and thus did not arise from the neoplastic clone. This low frequency of normal CFU-E was found in cultures containing $5.0 \mathrm{U} \mathrm{ESF} / \mathrm{ml}$. Because $<20 \%$ of total colony growth occurred in cultures without added ESF the majority of colony-forming cells expressed in cultures containing ESF arose from the poly-

${ }^{3}$ J. Mladenovic, N. Lin, and J. W. Adamson. Unpublished observations. cythemia vera clone. The findings with BFU-E were confirmatory. Thus, further growth of neoplastic colony-forming cells required the addition of ESF to culture. Consequently, although the ability to form colonies in the absence of added ESF identifies members of the abnormal clone, it is not a characteristic of all of the neoplastic colony-forming cells and thus does not define the limits of the clone.

To monitor disease progression and to determine if the percentage of normal progenitors in circulation accurately reflects their frequency in the marrow, the prevalences of normal colony-forming cells from blood and marrow were compared directly. Similar percentages for both normal granulopoietic and erythropoietic committed stem cells were observed. Thus, although normal colony-forming cells in circulation have different cell cycle characteristics than those in the marrow (11) and, perhaps, circulating BFU-E may have a different capacity to synthesize fetal hemoglobin in culture (12), there appeared to be nothing that favored the circulation of normal or abnormal progenitors. Consequently, it should be possible to monitor changes in the overall frequency of normal colonyforming cells in such patients by serial cultures of peripheral blood mononuclear cells.

The G-6-PD marker allows study of the origin of the committed stem cells, thereby providing the opportunity to investigate early commitment events in hematopoiesis. Commitment or restriction events are those that occur in pluripotent stem cells and lead to a restriction in the differentiation program that such a progenitor can express. Correlation analyses of various colony-forming cells in the mouse demonstrated that primitive BFU-E and CFU-C are closely related to the pluripotent stem cell (7). In our studies, we wished to determine whether distortions in the ratios of one progenitor type to another characterized the neoplastic clone as compared to the residual normal clones. If strikingly different ratios were found for the neoplastic clone, such differences might provide an explanation for the clinical phenotype of the disease. Thus, an increase in the number of BFU-E relative to CFU-C might characterize polycythemia vera, a disease 
clinically dominated by the increased erythrocyte mass, whereas a marked increase in CFU-C might characterize chronic myelogenous leukemia, a disease clinically dominated by increased granulopoiesis. On the other hand, if ratios of CFU-C:BFU-E were similar within the neoplastic and residual normal clones, it would be consistent with the contention that early restriction events in hematopoietic differentiation were not subject to regulatory control but, in fact, occurred in a stochastic fashion $(13,14)$. The ratios of CFU-C:BFU-E in cultures from patient 1 (3.2 in the abnormal clone and 2.7 in the residual normal clones) were not significantly different and well within our range established from 13 normal subjects. From patient 2, no normal colony-forming cells were detected. Thus, all data reflect the abnormal clone and the ratio was 2.0. Consequently, whereas the ratios of early progenitors may vary somewhat from patient to patient, no dramatic differences were found between normal subjects and the abnormal clones represented by the patients studied here.

These results are comparable to the ratios that can be calculated from the recent report by Eaves and Eaves (15) for chronic myelogenous leukemia. The mean ratio of CFU-C:primitive BFU-E estimated from the data on their three patients is $3.5(\mathrm{SEM} \pm 0.6)$. This value is similar to the results from the patients with polycythemia vera and from our normal controls. The similarity of these ratios suggests that while there may be increased numbers of abnormal pluripotent or committed stem cells in patients with chronic myeloproliferative disorders, the relative rates at which restriction to the erythroid or granulopoietic pathways occurs do not differ significantly from normal. Consequently, in most patients the clinical phenotypes of these diseases appear to depend upon differences in the regulatory control of cells that appear later in the maturation process than CFU-C and BFU-E. These differences must therefore lie in processes that lead to the amplification of the numbers of mature progeny.

Finally, this study allows a comparison of the prevalence of normal progenitors over time to determine whether disease progression is associated with changes in the frequency of normal colony-forming cells. In both patients, there was a significant decline in the frequency of normal colony-forming cells. While culture conditions clearly cannot be identical from year to year, similar declines in the percentage of normal CFU-C and BFU-E were observed for patient 1 over the interval and repeat studies in 1978 and 1979 confirmed this decline for both patients.

Originally, it was considered that such findings possibly represented a partial or complete extinction of residual normal stem cells by the neoplastic clone, extreme dilution of normal progenitors by abnormal ones, or suppression of normal stem cell differentia- tion at a more primitive level than CFU-C and BFU-E. A recent study in a G-6-PD heterozygote treated aggressively with chemotherapy and splenectomy for chronic myelogenous leukemia suggests that complete extinction of normal stem cells does not occur (16). In that patient, extensive treatment led to the re-emergence of normal colony-forming cells and almost total loss of the Philadelphia chromosome abnormality. Consequently, normal stem cells had to persist in that patient although they and their committed progeny were not detected in culture immediately before treatment. The recent development of assays for even more primitive progenitors in man $(17,18)$ hopefully will provide the opportunity to characterize the ratios of normal to abnormal stem cells in these diseases over time and determine whether a decline in the frequency of detectable normal stem cells has any prognostic or therapeutic importance.

\section{ACKNOWLEDGMENTS}

Portions of this work were supported by grants AM-19410, CA-18029, CA-16448, GM-15253, and CA-15704 from the Institute of Arthritis, Metabolism and Digestive Diseases, the Institute of General Medical Sciences, the National Cancer Institute, National Institutes of Health, and designated research funds of the Veterans Administration. A portion of this work was conducted through the Clinical Research Facility of the University of Washington, supported by the National Institutes of Health (grant RR-37).

\section{REFERENCES}

1. Adamson, J. W., P. J. Fialkow, S. Murphy, J. F. Prchal, and L. Steinmann. 1976. Polycythemia vera: stem-cell and probable clonal origin of the disease. N. Engl. J. Med. 295: 913-916.

2. Fialkow, P. J. 1973. Primordial cell pool size and lineage relationships of five human cell types. Ann. Hum. Genet. 37: 39-48.

3. Fialkow, P. J., S. M. Gartler, and A. Yoshida. 1967. Clonal origin of chronic myelocytic leukemia in man. Proc. Natl. Acad. Sci. U. S. A. 58: 1468-1471.

4. Fialkow, P. J., R. J. Jacobson, and T. Papayannopoulou. 1977. Chronic myelocytic leukemia: clonal origin in a stem cell common to the granulocyte, erythrocyte, platelet, and monocyte/macrophage. Am.J. Med. 63: 125-130.

5. Jacobson, R. J., A. Salo, and P. J. Fialkow. 1978. Agnogenic myeloid metaplasia: a clonal proliferation of hematopoietic stem cells with secondary myelofibrosis. Blood. 51: 189-194.

6. Prchal, J. F., J. W. Adamson, S. Murphy, L. Steinmann, and P. J. Fialkow. 1978. Polycythemia vera. The in vitro response of normal and abnormal stem cell lines to erythropoietin. J. Clin. Invest. 61: 1044-1047.

7. Gregory, C. J., and R. M. Henkelman. 1978. Relationships between early hemopoietic progenitor cells determined by correlation analysis of their numbers in individual spleen colonies. In Experimental Hematology Today. S. J. Baum and G. D. Ledney, editors. Springer-Verlag New York, Inc., New York. 93-101.

8. Eaves, C. J., and A. C. Eaves. 1979. Erythroid progenitor cell numbers in human marrow - implications for regulation. Exp. Hematol. 7(Suppl. 5): 54-64. 
9. Singer, J. W., P. J. Fialkow, L. W. Dow, C. Ernst, and L. Steinmann. 1979. Unicellular or multicellular origin of human granulocyte-macrophage colonies in vitro. Blood. 54: $1395-1399$.

10. Eaves, C. J., and A. C. Eaves. 1978. Erythropoietin (Epo) dose-response curves for three classes of erythroid progenitors in normal human marrow and in patients with polycythemia vera. Blood. 52: 1196-1210.

11. Tebbi, K., S. Rubin, D. H. Cowan, and E. A. McCulloch. 1976. A comparison of granulopoiesis in culture from blood and marrow cells of nonleukemic individuals and patients with acute leukemia. Blood. 48: 235-243.

12. Housman, D., B. Clarke, D. Hillman, B. Alter, B. Forget, and D. Nathan. 1979. Control of fetal hemoglobin synthesis in adult erythroid precursor cells. Evidence for different programs for bone marrow and peripheral blood precursors. In Cellular and Molecular Regulation of Hemoglobin Switching. G. Stamatoyannopoulos and A.W. Nienhuis, editors. Grune \& Stratton, Inc., New York. 351-366.

13. Till, J. E., E. A. McCulloch, and L. Siminovich. 1964. A stochastic model of stem cell proliferation based on the growth of spleen colony-forming cells. Proc. Natl. Acad. Sci. U. S. A. 51: 29-35.

14. Korn, A. P., R. M. Henkelman, F. P. Ottensmeyer, and J. E. Till. 1973. Investigations of a stochastic model of haemopoiesis. Exp. Hematol. 1: 362-375.

15. Eaves, A. C., and C. J. Eaves. 1979. Abnormalities in the erythroid progenitor compartments in patients with chronic myelogenous leukemia. Exp. Hematol. 7(Suppl. 5): $65-75$.

16. Singer, J. W., Z. Arlin, V. Najfeld, J. W. Adamson, F. Kempin, B. Clarkson, and P. J. Fialkow. 1980. Restoration of non-clonal, presumably normal hematopoiesis accompanying a chemotherapeutic conversion of $\mathrm{Ph}^{1}$-positive chronic myelogenous leukemia (CML) to $\mathrm{Ph}^{1}$-negative. Blood. 56: 356-360.

17. Fauser, A. A., and H. A. Messner. 1978. Granulo-erythropoietic colonies in human bone marrow, peripheral blood, and cord blood. Blood. 52: 1243-1247.

18. Fauser, A. A., and H. A. Messner. 1979. Identification of megakaryocytes, macrophages, and eosinophils in colonies of human bone marrow containing neutrophilic granulocytes and erythroblasts. Blood. 53: 1023-1027. 